\title{
Toward Novel Wearable Pyroelectric Temperature Sensor for Medical Applications
}

\author{
Alan Davidson, Arjan Buis, and Ivan Glesk, Senior Member, IEEE
}

\begin{abstract}
Knowledge of an amputees' residual limb skin temperature is considered to be of particular importance as an indicator of tissue health. Temperature within the prosthetic socket typically varies over the range $25^{\circ} \mathrm{C}$ to $35^{\circ} \mathrm{C}$ and this warm, confined environment causes sweating which creates favourable conditions for both the growth of bacteria and an increased risk of tissue breakdown. With this in mind a wearable sensor for the real-time measurement of temperature variations at the prosthetic socket/liner interface is under development and a proof of concept prototype is presented. The sensor exploits the large pyroelectric effect present in ferroelectric lead zirconate titanate $\mathrm{Pb} \mathrm{Zr}_{\mathrm{x}}\left(\mathrm{Ti}_{(1-\mathrm{x})}\right) \mathbf{O}_{3}$ (PZT) and has several inherent advantages over other methods of temperature sensing. The sensing element is a low cost commercially available thick-film PZT device. Mathematical models are developed to describe the sensor immitance and response to temperature change, and both the clamped and unclamped capacitances are investigated over the range $20^{\circ} \mathrm{C}$ to $40^{\circ} \mathrm{C}$. Sensor characteristics were found to be dominated by the clamped dielectric constant and operation under short-circuit conditions is found to offer a constant sensor gain over the temperature range of interest
\end{abstract}

Index Terms-e-Health, Piezoelectric, Pyroelectric, Temperature Sensor, Sensor model analysis

\section{INTRODUCTION}

$\mathrm{T}$ HE use of even a well-fitting prosthesis by a health impaired or otherwise healthy person with a lower limb amputation can result in the increased risk of tissue breakdown, infection and decubitus (pressure) ulcers [1]. In particular, it is suggested that raised temperature is associated with the risk of tissue maceration and infection through a combination of confined environment and perspiration [2].

This paragraph of the first footnote will contain the date on which you submitted your paper for review. It will also contain support information, including sponsor and financial support acknowledgment. For example, "This work was supported in part by the U.S. Department of Commerce under Grant BS123456".

The next few paragraphs should contain the authors' current affiliations, including current address and e-mail. For example, F. A. Author is with the National Institute of Standards and Technology, Boulder, CO 80305 USA (email: author@ boulder.nist.gov).

S. B. Author, Jr., was with Rice University, Houston, TX 77005 USA. He is now with the Department of Physics, Colorado State University, Fort Collins, CO 80523 USA (e-mail: author@ lamar.colostate.edu).

T. C. Author is with the Electrical Engineering Department, University of Colorado, Boulder, CO 80309 USA, on leave from the National Research Institute for Metals, Tsukuba, Japan (e-mail: author@nrim.go.jp).
Furthermore it has been proposed that a localised increase in temperature may be an indicator of an increase in pressure induced deep tissue injury (DTI) with DTI in turn being clinically associated with the development of decubitus ulcers [3]. It is therefore desirable to constantly monitor temperature within the socket to enable the wearer and relevant health authority to be provided with an early warning of the development of potentially unhealthy conditions. Such a sensor system must be wearable, lightweight, reliable and robust. In addition the sensor must not interfere with the functioning of the prosthetic socket and liner.

In the selection of a temperature sensor technology the following requirements of the sensing element and signal conditioning were identified:

- Have a low thickness profile at the sensor site

- Exhibit no self-heating at the sensor site

- Be portable and operate from a $5 \mathrm{v}$ low current rating power supply

- Have low power consumption and require minimal wiring

- Be low cost, rugged and exhibit linearity and repeatability

- Require no temperature reference

Thermocouples are rugged, inexpensive and are selfpowered; however the requirement of a stable reference temperature is problematic for a wearable sensor which will be exposed to a constantly changing environment. The cold junction compensation method requires the measurement of temperature by another method which would increase the cost, weight and complexity. Resistive temperature detectors (RTD's) are more stable than thermocouples and provide a high degree of accuracy and linearity. In addition, RTD's are also available with a low thickness profile where a thin film of conductor is deposited on a ceramic substrate. However RTD's are expensive, fragile and have relatively high power consumption. In addition they require a current source and are prone to inaccuracy due to self-heating. Thermistors are relatively inexpensive in comparison to RTD's but are prone to the same disadvantages, in addition to having a non-linear response.

In light of these shortcomings, detailed consideration was given to the utilisation of the pyroelectric effect inherent in PZT. The pyroelectric effect is widely employed in pyrometry 
for remote temperature sensing, infrared imaging and motion detection [4],[5] and [6]. When operated within their linear response regime, that is where the response is small enough that the response is directly proportional to an applied stimulus, PZT devices provide an interesting proposition as they satisfy all of the requirements highlighted above. PZT devices are widely used for sensor, resonator and actuator applications and have among their advantages a relatively large charge sensitivity, high Curie temperature, high coupling coefficient and low cost.

In particular, as a sensor the PZT devices are self-powered, do not self-heat and are both rugged and have a low thickness profile. In common with all pyroelectric and piezoelectric devices they exhibit dielectric loss which at low frequency and DC is due to leakage currents [7]. With a typical resistivity of the order $10^{10} \Omega-\mathrm{m}$ at room temperature, they cannot therefore be used for DC temperature measurements. The DC response voltage will decay exponentially with a time constant equal to $\mathrm{RC}$ where $\mathrm{R}$ and $\mathrm{C}$ are the leakage resistance and capacitance of the device respectively. However with good signal conditioning design it is possible to realise long time constants making it possible to measure pseudo-static and continuous very low frequency temperature variations about a mean.

The ultimate goal is to find a very low cost solution that in tandem with the appropriate signal conditioning will serve the dual purpose of measuring both residual limb temperature and interface pressure. A crucial advantage of using ferroelectrics such as PZT is that in addition to temperature, this technology is equally suitable for measuring interface pressure through the direct piezoelectric effect where an electric field and electric displacement are generated in response to an applied stress. This is of particular interest as in common with temperature, interface pressure is also clinically accepted as affecting tissue health and is implicated in the development of decubitus ulcers [8].

A prototype temperature sensor based on the pyroelectric effect has been designed to provide continuous measurement of temperature within a prosthetic socket. In operation it is envisaged that the sensor would be recessed into the socket wall using a compliant adhesive to allow free thermal expansion and to avoid erroneous responses due to strains induced by socket flexing.

The temperature sensing element is a commercially available Murata (Type 7BB-27-4) diaphragm consisting of a PZT thick film poled along the 3-axis normal to the surface with silver electrodes on the top and bottom faces perpendicular to the axial direction (3-axis) and bonded to a brass substrate. The type of PZT is not known nor is this information available from Murata. However it is likely that the material used is PZT5H commonly used for this type of application. The manufacturer specified flexure mode resonant frequency is $4.6 \mathrm{kHz}$ with a low frequency capacitance of $20 \mathrm{nF} \pm 30 \%$ measured at $1 \mathrm{kHz}$. Using electronic callipers, the diameters of the PZT film and electrode are $20 \mathrm{~mm}$ and $18.2 \mathrm{~mm}$ respectively. The total thickness is $0.53 \mathrm{~mm}$ made up of a $0.3 \mathrm{~mm}$ brass substrate and $0.23 \mathrm{~mm}$ PZT film. While the
PZT based technology described in this paper has the inherent advantage of being appropriate to both temperature and interface pressure measurement, this paper focuses specifically on temperature measurement by taking advantage of the large inherent constant strain pyroelectric coefficient of PZT materials. This high pyroelectric coefficient in tandem with appropriate signal conditioning allows the measurement of temperature variations in the $\mathrm{uHz}$ and $\mathrm{mHz}$ range observed within the confinement of a prosthetic socket [9]. It is evident from the analysis described below that there are additional secondary pyroelectric effect contributions to the response due to the piezoelectric effect where thermal expansion induces stress and strain in both the PZT film and substrate. In the absence of the brass substrate the total pyroelectric response is due to the constant stress pyroelectric coefficient which is the sum of the primary and secondary pyroelectric effects.

The temperature within a transtibial prosthetic socket typically varies within the range $25^{\circ} \mathrm{C}$ to $35^{\circ} \mathrm{C}$ [10] and it is therefore necessary to investigate the temperature dependence of the sensor capacitance over this temperature range. The sensor capacitance is in turn dependent on the clamped permittivity and piezoelectric constants of the PZT; and stiffness matrix elements of both PZT and substrate. The pyroelectric current generated is also dependent on the piezoelectric constants and stiffness matrix elements of both PZT and substrate in addition to the primary constant strain pyroelectric coefficient and the coefficients of thermal expansion. The inherent temperature dependence of these material constants may therefore significantly affect the response of the sensor by influencing the low frequency capacitance of the sensing element.

It is assumed that the brass substrate is rigidly bonded to the PZT film bottom electrode using a conductive epoxy resin adhesive, and furthermore that the maximum temperature the sensor will be subjected to is below that of the adhesive glass transition temperature such that the adhesive remains in its vitreous form. The effect of the adhesive can be justifiably ignored by the following argument: The coefficient of thermal expansion of the adhesive below the glass transition temperature is typically $50 \%$ greater than that of the brass substrate. However the thickness and Young's modulus of the adhesive layer (typically around 20um and 6Gpa respectively) are much lower than that of the substrate and PZT film. An estimate based on the above values indicates that the effective stress strain ratio in the 1- and 2-axes of the PZT film and effective coefficient of thermal expansion of the brass substrate including the effect of the adhesive layer differ from those where the adhesive layer is neglected by less than $0.2 \%$ and $0.3 \%$ respectively.

The temperature dependence of the PZT element capacitance is investigated using both low frequency and resonant frequency impedance measurements over the temperature range $20^{\circ} \mathrm{C}$ to $40^{\circ} \mathrm{C}$. A low frequency impedance model allows the analysis of the low frequency capacitance while resonance methods are used to measure the clamped capacitance of the sensor using the well-known Butterworth 
Van Dyke resonator model. In addition, voltage and current response models are developed to describe the response of the sensor element to temperature change under substrate clamping conditions. The models are developed on the basis of assuming a rectilinear form however the analysis can also be extended to sensing elements with an annular form by considering the tangential and radial components of stress and strain in a cylindrical coordinate system.

\section{THEORETICAL BACKGROUND}

Equations (1) and (2) are defined as the linear piezoelectric constitutive equations in matrix stress-charge form that describe the coupled dielectric-elastic-thermal behaviour of the sensor element.

$T=c^{E, \tau} S-e^{t, \tau} E-\gamma^{E} \theta$

$D=e^{\tau} S+\varepsilon^{s, \tau} E+P^{s} \theta$

Where $\mathrm{T}$ is the stress $\left(\mathrm{N} / \mathrm{m}^{2}\right), c^{E, \tau}$ is the stiffness, $S$ is the strain $(\mathrm{m} / \mathrm{m}), e^{t, \tau}$ is the piezoelectric stress-charge coefficient $\left(\mathrm{C} / \mathrm{m}^{2}\right), E$ is the electric field $(\mathrm{V} / \mathrm{m}), \theta$ is the small temperature change $(\mathrm{K}), D$ is the electric displacement $\left(\mathrm{C} / \mathrm{m}^{2}\right), \varepsilon^{s, \tau}$ is the constant strain permittivity, $P^{s}$ is the constant strain pyroelectric coefficient $\left(\mathrm{C} / \mathrm{m}^{2} \mathrm{~K}\right)$ and $\gamma^{E}$ is the thermal stress coefficient $\left(\mathrm{N} / \mathrm{m}^{2} \mathrm{~K}\right)$ equal to $c^{E, \tau} \varphi_{p}$ where $\varphi_{p}$ is the coefficient of thermal expansion $(\mathrm{m} / \mathrm{mK})$ matrix. The superscripts $\tau, E$ and $S$ indicate isothermal conditions, constant electric field and constant strain respectively while the superscript $t$ indicates transpose matrix. To reduce notation the superscript $\tau$ is henceforth omitted.

Equations (1) and (2) are valid at DC and low frequencies where the wavelength of the mechanical disturbance in each axis is very much greater than the dimensions of the sensor. The term $P^{s} \theta$ represents the primary pyroelectric effect and $\gamma^{E} \theta$ the secondary pyroelectric effect. In general the primary effect produces a significantly greater response to temperature change than the secondary effect.

The stress and strain in the 1 and 2 directions parallel to the surface of the sensor under substrate clamping conditions are equal such that $T_{1}=T_{2}=T$ and $S_{1}=S_{2}=S$ and the transverse isotropic nature of PZT means that $c_{31}^{E}=$ $c_{32}^{E}=c_{13}^{E}=c_{23}^{E}, c_{12}^{E}=c_{21}^{E}, c_{11}^{E}=c_{13}^{E}$ and $e_{31}=e_{32}$. Under these conditions, expansion of the matrix equations (1) and (2) for poling in the 3-direction perpendicular to the surface of the sensor yields (3) to (5).

$$
\begin{aligned}
& T=\left(c_{11}^{E}+c_{12}^{E}\right)\left(S-\varphi_{p 1} \theta\right)+c_{31}^{E}\left(S_{3}-\varphi_{p 3} \theta\right)-e_{31} E_{3} \\
& T_{3}=2 c_{31}^{E}\left(S-\varphi_{p 1} \theta\right)+c_{33}^{E}\left(S_{3}-\varphi_{p 3} \theta\right)-e_{33} E_{3} \\
& D_{3}=2 e_{31} S+e_{33} S_{3}+\varepsilon_{33}^{S} E_{3}+P^{s} \theta
\end{aligned}
$$

In contrast to the PZT film, the brass substrate is an alloy and it is therefore assumed to behave as an isotropic material.
For the case that the stress and strain in the 1- and 2- axes of the brass substrate are equal such that $T_{b 1}=T_{b 2}=T_{b}$ and $S_{b 1}=S_{b 2}=S_{b}$, and that the stress in the 3-axis is zero $\left(T_{b 3}=0\right)$, then Hooke's law for the substrate under varying temperature conditions is represented by (6). The subscript $b$ indicates a quantity that applies to the brass substrate.

$T_{b}=\frac{E_{b}}{\left(1-\mu_{b}\right)}\left(S_{b}-\varphi_{b} \theta\right)$

The material constants $\varphi_{\mathrm{b}}, \mu_{b}$ and $\mathrm{E}_{\mathrm{b}}$ are the coefficient of thermal expansion, Poisson's ratio and Young's modulus of the substrate respectively. If the PZT film and substrate are now firmly bonded together, then the strain along the 1- and 2axes are equal in both the PZT film and brass substrates over the area of contact, i.e. $S=S_{b}$. In addition, since the forces within each material must sum to zero, then $T_{b} A_{b}+T A_{p}=0$, where $A_{b}$ and $A_{p}$ are the cross-sectional areas of the brass substrate and PZT film respectively in the 1-2 and 1-3 planes. On the assumption that the resulting bi-layer composite remains flat over the temperature range of interest i.e. the radius of curvature remains close to infinite and therefore has a negligible effect under thermal expansion, the stress along the 1- and 2-axes in the PZT film is then given by (7).

$T=-\frac{E_{b}}{\left(1-\mu_{b}\right)} \frac{A_{b}}{A_{p}}\left(S-\varphi_{b} \theta\right)$

Equations (3) through (5) and (7) can be used under different boundary conditions to determine models for the low frequency immitances, open circuit output voltage via $E_{3}$ and short-circuit current via $D_{3}$.

\section{SENSOR MODELLING}

\section{A. Open-circuit sensor model}

Given that there is no applied stress in the 3-direction or flow of current between the device electrodes the boundary conditions for the open-circuit model are $T_{3}=0$ and $D_{3}=0$ with $T$ given by (7). Equations (3) to (5) therefore become:

$$
\begin{aligned}
& -\frac{E_{b}}{\left(1-\mu_{b}\right)} \frac{A_{b}}{A_{p}}\left(S-\varphi_{b} \theta\right)=\left(c_{11}^{E}+c_{12}^{E}\right)\left(S-\varphi_{p 1} \theta\right) \\
& +c_{31}^{E}\left(S_{3}-\varphi_{p 3} \theta\right)-e_{31} E_{3}
\end{aligned}
$$

Where $\varphi_{p 1}$ and $\varphi_{p 3}$ are the coefficients of thermal expansion of the PZT in the 1- \& 2-axes and 3-axis respectively. After manipulation of (8) and (9), $S$ and $S_{3}$ can be found in terms of $E_{3}$. Substitution of these expressions into (10) and rearranging for $E_{3}$ yields (11) describing the electric field response to a change in sensor temperature. 

HERE TO EDIT) <

$E_{3}=-\frac{\frac{\beta\left(\varphi_{b}-\varphi_{p 1}\right) E_{b} A_{b}}{E_{b} A_{b}+\alpha\left(1-\mu_{b}\right) A_{p}}+\left(2 \varphi_{p 1} e_{31}+\varphi_{p 3} e_{33}\right)+P^{s}}{\frac{\left(1-\mu_{b}\right) \beta^{2} A_{p}}{2\left(E_{b} A_{b}+\alpha\left(1-\mu_{b}\right) A_{p}\right)}+\frac{e_{33}^{2}}{c_{33}^{E}}+\varepsilon_{33}^{s}} \theta$

Where $\alpha=c_{11}^{E}+c_{12}^{E}-2 \frac{\left(c_{31}^{E}\right)^{2}}{c_{33}^{E}}$ and $\beta=2\left(e_{31}-e_{33} \frac{c_{31}^{E}}{c_{33}^{E}}\right)$

An effective pyroelectric coefficient $P_{\text {eff }}$ can therefore be defined as the sum of the constant stress pyroelectric coefficient $\left(2 \varphi_{p 1} e_{31}+\varphi_{p 3} e_{33}\right)+P^{s}$ and an additional term due to substrate clamping. The contribution to $P_{\text {eff }}$ of each term in the numerator of (11) will be discussed in section V.

Since $E_{3}$ can be considered constant with dimension along the 3 -axis, the output voltage is given by (12).

$V_{\text {out }}=-\int_{0}^{l_{p}} E_{3} d x_{3}=-l_{p} E_{3}$

Where $x_{3}$ represents the 3 -axis and $l_{p}$ is the thickness of the PZT film. The negative sign is a result of the generation of a negative potential gradient across the electrodes of the piezoelectric film with respect to $E_{3}$. The output voltage is therefore given by (13).

$V_{\text {out }}=l_{p} \frac{\frac{\beta\left(\varphi_{b}-\varphi_{p 1}\right) E_{b} A_{b}}{E_{b} A_{b}+\alpha\left(1-\mu_{b}\right) A_{p}}+\left(2 \varphi_{p 1} e_{31}+\varphi_{p 3} e_{33}\right)+P^{s}}{\frac{\left(1-\mu_{b}\right) \beta^{2} A_{p}}{2\left(E_{b} A_{b}+\alpha\left(1-\mu_{b}\right) A_{p}\right)}+\frac{e_{33}^{2}}{c_{33}^{E}}+\varepsilon_{33}^{s}} \theta$

$\mathrm{V}_{\text {out }}$ can therefore be interpreted as due to a bound charge density $Q\left(\mathrm{C} / \mathrm{m}^{2}\right)$ applied to a capacitance $C_{t}$ where $Q$ is generated by a temperature induced change in polarisation due to both the primary and secondary pyroelectric effects enhanced by the influence of the substrate. The expressions for $Q$ and $C_{t}$ are given by (15) and (16) where A is the surface area of the PZT film top electrode. Equation (14) describes the constant stress capacitance, $C^{T}$ :

$C^{T}=\frac{A \varepsilon_{33}^{T}}{l_{p}}=\frac{A}{l_{p}}\left(\frac{\beta^{2}}{2 \alpha}+\frac{e_{33}^{2}}{c_{33}^{E}}+\varepsilon_{33}^{s}\right)$

Where $\varepsilon_{33}^{T}$ is the constant stress permittivity and $C^{T}$ describes the capacitance of a sensor element free to strain in all 3 axes. The effect of substrate clamping is therefore to reduce the capacitance $C^{T}$ to $C_{t}$ described by (16).

$Q=\left(\frac{\beta\left(\varphi_{b}-\varphi_{p 1}\right) E_{b} A_{b}}{E_{b} A_{b}+\alpha\left(1-\mu_{b}\right) A_{p}}+\left(2 \varphi_{p 1} e_{31}+\varphi_{p 3} e_{33}\right)+P^{s}\right) \theta$

$C_{t}=\frac{A}{l_{p}}\left(\frac{\left(1-\mu_{b}\right) \beta^{2} A_{p}}{2\left(E_{b} A_{b}+\alpha\left(1-\mu_{b}\right) A_{p}\right)}+\frac{e_{33}^{2}}{c_{33}^{E}}+\varepsilon_{33}^{S}\right)$

The low frequency capacitance of the sensor $C_{t}$ is therefore the parallel sum of the electrostatic clamped (or constant strain) capacitance, $C_{o}=A \varepsilon_{33}^{S} / l_{p}$ and an electrical equivalent mechanical capacitance $C_{m}$ given by (17).
$C_{m}=\frac{A}{l_{p}}\left(\frac{\left(1-\mu_{b}\right) \beta^{2} A_{p}}{2\left(E_{b} A_{b}+\alpha\left(1-\mu_{b}\right) A_{p}\right)}+\frac{e_{33}^{2}}{c_{33}^{E}}\right)$

It is clear that, if the material constants have a significant temperature dependency, then subsequently $C_{t}$ will vary with temperature, and $Q$ and the sensor response will be non-linear with temperature. The output voltage will be dependent not only on the desired pyroelectric effects but also on temperature dependent changes in the material constants of both the PZT film and brass substrate.

\section{B. Short-circuit model}

The short-circuit model can be derived directly from (13) by replacing $\varepsilon_{33}^{S}$ with a complex clamped permittivity $\boldsymbol{\varepsilon}_{33}^{\boldsymbol{S}}=\varepsilon_{33}^{S}-j \sigma / \omega$ where bold script indicates a complex quantity, $\sigma$ is the effective conductivity $(\mathrm{S} / \mathrm{m})$ of the PZT film and $\omega$ is the frequency ( $\mathrm{rad} / \mathrm{s})$. This substitution is normally used to represent dielectric leakage however it is also useful for the inclusion of an additional parallel impedance across the sensor electrodes to represent the input impedance of the signal conditioning electronics.

Given the simple geometry of the sensor, $\sigma$ can be replaced by $\sigma=l_{p} / R A$, where $R$ is a notional resistance connected across the electrodes of the PZT film. The current through $\mathrm{R}$ is given by $I_{\text {out }}=V_{\text {out }} / R$ and $I_{\text {out }}$ is related to $D_{3}$ by $I_{\text {out }}=$ $j \omega A D_{3}$. Replacing $\varepsilon_{33}^{S}$ in (13) with $\varepsilon_{33}^{S}-j \sigma / \omega$ and substituting $l_{p} / R A$ for $\sigma$ results in (17) for $I_{\text {out }}$.

$I_{\text {out }}=l_{p} \frac{\frac{\beta\left(\varphi_{b}-\varphi_{p 1}\right) E_{b} A_{b}}{E_{b} A_{b}+\alpha\left(1-\mu_{b}\right) A_{p}}+\left(2 \varphi_{p 1} e_{31}+\varphi_{p 3} e_{33}\right)+P^{S}}{R\left(\frac{\left(1-\mu_{b}\right) \beta^{2} A_{p}}{2\left(E_{b} A_{b}+\alpha\left(1-\mu_{b}\right) A_{p}\right)}+\frac{e_{33}^{2}}{c_{33}^{E}}+\varepsilon_{33}^{s}\right)+l_{p} / j \omega A} \theta$

As $R$ is reduced, $E_{3}$ reduces and the sensor response becomes less dependent on $\varepsilon_{33}^{S}$. In the limit as $R \rightarrow 0$, the influence of $\varepsilon_{33}^{s}$ vanishes while the response remains dependent on the remaining material constants. In practice $R$ will be the parallel combination of the input impedance of the signal conditioning amplifier and the leakage resistance of the PZT film. In the case of charge mode signal conditioning, $R$ is dominated by the low input impedance of the amplifier and results in a cut-off frequency equal to $1 / 2 \pi R C_{t}$. Since $R$ is small, this cut-off frequency is generally high such that it has a vanishingly small effect on the low frequency response of the sensor. In the limit as $R \rightarrow 0$, (18) reduces to (19) giving the short circuit current. The associated electric displacement is given by (20).

$$
\begin{aligned}
& I_{s / c}=j \omega A\left(\frac{\beta\left(\varphi_{b}-\varphi_{p 1}\right) E_{b} A_{b}}{E_{b} A_{b}+\alpha\left(1-\mu_{b}\right) A_{p}}+\left(2 \varphi_{p 1} e_{31}+\varphi_{p 3} e_{33}\right)+P^{s}\right) \theta \\
& D_{s / c}=\left(\frac{\beta\left(\varphi_{b}-\varphi_{p 1}\right) E_{b} A_{b}}{E_{b} A_{b}+\alpha(1-\gamma) A_{p}}+\left(2 \varphi_{p 1} e_{31}+\varphi_{p 3} e_{33}\right)+P^{s}\right) \theta
\end{aligned}
$$

Equation (20) represents the free charge density induced by a temperature change $\theta$ which is numerically equal to $Q$. The 

HERE TO EDIT) <

free charge flowing between the sensor element electrodes is therefore given by $A D_{s / c}$.

\section{Immitance modelling}

To investigate the effect of temperature on the performance of the sensor an immitance model is presented to describe the low frequency capacitance $C_{t}$. In the low frequency regime the effect of the inertial masses of the brass substrate and PZT body can be neglected. The clamped capacitance $C_{0}$ is obtained using the Butterworth Van Dyke (BVD) piezoelectric resonator model. The electrical equivalent mechanical capacitance $C_{m}$ can then be obtained from $C_{m}=C_{t}-C_{0}$. Under the conditions that $T_{3}=0$ and that the measurement temperature is held constant over the period of measurement such that $\theta=0$ then (3) to (5) reduce to (20) to (22).

$-\frac{E_{b}}{\left(1-\mu_{b}\right)} \frac{A_{p}}{A_{b}} S=\left(c_{11}^{E}+c_{12}^{E}\right) S+c_{31}^{E} S_{3}-e_{31} E_{3}$

$0=2 c_{31}^{E} S+c_{33}^{E} S_{3}-e_{33} E_{3}$

$D_{3}=2 e_{31} S+e_{33} S_{3}+\varepsilon_{33}^{S} E_{3}$

By algebraic manipulation of (21) and (22) both $S$ and $S_{3}$ can be found in terms of $E_{3}$. Further substitution into (23) results in:

$\frac{D_{3}}{E_{3}}=\frac{\left(1-\mu_{b}\right) \beta^{2} A_{p}}{2\left(E_{b} A_{b}+\alpha\left(1-\mu_{b}\right) A_{p}\right)}+\frac{e_{33}^{2}}{c_{33}^{E}}+\varepsilon_{33}^{S}$

For an applied voltage $\mathrm{V}$ that gives rise to a current $I$, and noting that $D_{3}=-I / j \omega A$, and that for the case of an applied electric field, $E_{3}=-V / l_{p}$ then the low frequency admittance $Y_{l f}=I / V$ is given by (24).

$Y_{l f}=\frac{j \omega A}{l_{p}}\left[\frac{\left(1-\mu_{b}\right) \beta^{2} A_{p}}{2\left(E_{b} A_{b}+\alpha\left(1-\mu_{b}\right) A_{p}\right)}+\frac{e_{33}^{2}}{c_{33}^{E}}+\varepsilon_{33}^{s}\right]$

$Y_{l f}$ can be written $Y_{l f}=G_{l f}+j B_{l f}$ where $G_{l f}$ is the low frequency conductance (S) and $B_{l f}$ is the low frequency susceptance (S). When (25) is compared with (16) it is apparent that the measured low frequency susceptance $B_{l f}$ is equal to $\omega C_{t}$ and that $C_{t}$ can therefore be obtained by measuring the gradient of a $B_{l f}-\omega$ plot. In the derivation of $Y_{l f}$ losses are considered to be negligible and therefore the admittance is purely susceptive. This is a reasonable assumption in the low frequency regime however if required, mechanical and electrical losses can be introduced by treating the material constants as complex, resulting in an additional real valued conductance term.

When excited by an AC signal, the sensor becomes a resonator that may be described using the well-known BVD model shown in Fig. 1 which is valid only for a narrow range of frequencies around a resonance. $R_{s}, C_{s}$ and $L_{s}$ form the mechanical arm and account for mechanical damping, stiffness and inertial mass respectively. While the value and interpretation of $R_{S}, L_{s}$ and $C_{S}$ is dependent on resonance mode, clamping and mass loading on the sensor, the value of $C_{o}$ always represents the clamped capacitance $C_{o}=A \varepsilon_{33}^{s} / l_{p}$ of the sensor.

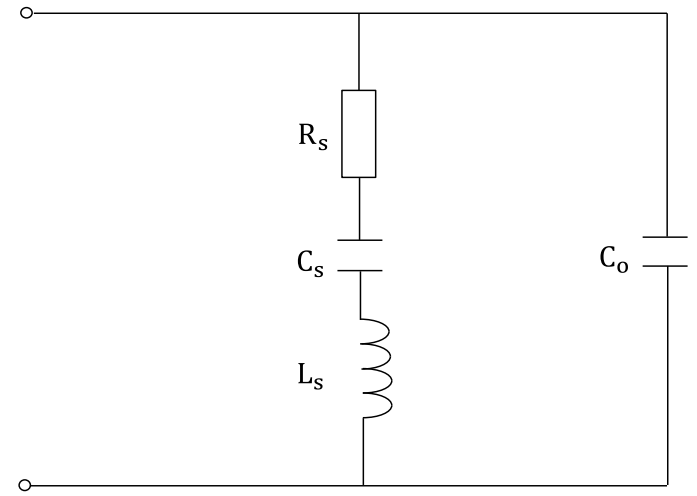

Fig. 1. BVD electrical equivalent impedance model of a piezoelectric resonator

Equation (26) describes the admittance $Y$ of the BVD model $Y=G+j B$ where $G$ is the conductance (S) and $B$ is the susceptance $(\mathrm{S})$.

$$
Y=\frac{R_{S}+j\left(\omega C_{0} R_{S}^{2}-\left(\omega L_{S}-\frac{1}{\omega C_{S}}\right)\left(1+\frac{C_{o}}{C_{S}}-\omega^{2} C_{0} L_{S}\right)\right)}{R_{S}^{2}+\left(\omega L_{S}-\frac{1}{\omega C_{S}}\right)^{2}}
$$

At series resonance, $\omega_{s}=1 / \sqrt{L_{s} C_{s}}$ and the conductance $\mathrm{G}$ reaches a peak value while the susceptance, $B$ is equal to $\omega_{s} C_{0}$ where $\omega_{\mathrm{s}}$ is the series resonance frequency. The capacitance measured at peak conductance is therefore the clamped capacitance $C_{0}$ of the piezoelectric sensor.

\section{MethodOLOGY}

\section{A. Immitance Measurement}

The temperature dependencies of the clamped capacitance $C_{0}$ and low frequency capacitance $C_{t}$ were investigated by measuring the impedance of the sensor isothermally over the frequency ranges $1 \mathrm{kHz}$ to $200 \mathrm{kHz}$ to include the first radial mode of vibration, and over the frequency range $1 \mathrm{kHz}$ to $3 \mathrm{kHz}$ respectively. The temperature was varied in steps of $5^{\circ} \mathrm{C}$ over the range $20^{\circ} \mathrm{C}$ to $40^{\circ} \mathrm{C}$. It was assumed that the elastic and electrical constants can be considered constant over the measured frequency range and that the resistivity of the electrodes is negligible.

The piezoelectric PZT film is very thin in comparison to its diameter and therefore radial modes are excited at frequencies below that of the thickness resonant modes. The first vibration resonance observed and used to determine $C_{0}$ is therefore radial. The use of the first radial vibration mode ensures a clean measurement uncorrupted by the coincidence of thickness mode and higher frequency radial mode resonances. The PZT element is supplied pre-fitted with aluminium flying leads. A $100 \mathrm{mv}$ ac signal was applied to the sensor using an Agilent B1500A impedance analyser connected via a 

HERE TO EDIT) <

Signatone probe station. The probe station was mounted on a vibration isolation table. The sensor lead terminations were fixed to a glass slide using Agar electrically conducting silver paste to form electrodes and connected to the impedance analyser via probes lowered onto the dried silver paste electrodes. The sensor was placed on a European Thermodynamics Peltier tile using RS Heat Sink Compound Plus paste to limit mechanical loading and ensure good thermal conduction over the entire brass substrate. The paste also served to effectively suppress the large amplitude $4.6 \mathrm{kHz}$ flexure mode ensuring uncorrupted low frequency imittance measurements. Temperature variations of the sensor element during impedance measurements due to room convection currents will result in the measurement of an additional pyroelectric current by the impedance analyser. This current is in addition to that generated by the impedance analyser and will therefore result in an error to the instantaneous impedance calculated. To minimise error, the sensor was shielded by placing the entire experimental set-up inside the probe station. The Peltier tile was fixed to an aluminium heat sink and connected to a KamView PID controller, with the temperature being selected using the KamView user interface. The Peltier tile temperature feedback signal was implemented by the use of a thermocouple attached to the Peltier tile surface. The risk of condensation on the sensor was avoided by ensuring that the ambient temperature was maintained below the minimum measurement temperature and that the Peltier temperature did not drop below ambient temperature during its hunting period. The temperature was increased in $5^{\circ} \mathrm{C}$ steps over a temperature range of $20^{\circ} \mathrm{C}$ to $40^{\circ} \mathrm{C}$. Prior to each measurement, the PZT element was manually short-circuited to ensure zero electric field and the temperature was allowed to stabilise for 15 minutes to avoid pyroelectric effects and to ensure a constant and uniform temperature throughout the body of the sensor.

\section{B. Response measurement}

The pyroelectric response of the sensor was measured in $2^{\circ} \mathrm{C}$ increments for a round trip over the temperature range $20^{\circ} \mathrm{C} \rightarrow 42^{\circ} \mathrm{C} \rightarrow 20^{\circ} \mathrm{C}$ using a compact portable differential input signal conditioning charge amplifier with the voltage response being proportional to the charge produced by the PZT sensor element. The sensor response was recorded using the charge amplifier and an Arduino Uno platform equipped with an HC-05 Bluetooth module which pushes the data over $\mathrm{WiFi}$ to a remote server by mobile phone. The voltage response was also measured using an Agilent DSO7052A oscilloscope. The response produced by the charge amplifier gradually decays with a time constant of approximately 30 minutes due to the capacitance and finite feedback resistance of the feedback network Since the duration of the measurement period was around 40 minutes, the continuous measurement of the temperature response was not possible due to the significant amplifier response decay over the course of the measurement period. It was therefore necessary to measure the response between each temperature increment separately. The procedure used was: 1) measurement of the steady state sensor response to a temperature increase of $20^{\circ} \mathrm{C}$ $-22^{\circ} \mathrm{C}$ is measured. 2) At $22^{\circ} \mathrm{C}$ the signal conditioning amplifier feedback networks are reset by discharging giving zero voltage at the amplifier output. 3) The steady state response of an increase of $22^{\circ} \mathrm{C}-24^{\circ} \mathrm{C}$ is measured. Steps 2) and 3) are repeated and so on. The response was measured at precisely the same interval of 2 minutes after initiation of each temperature increment to allow the PID controller time to settle at each temperature. The time taken between the initiation of each temperature increment and measurement of the response was maintained at precisely 3 minutes including the 2 minute settling time. An accumulative voltage can therefore be defined as the sum of the individual temperature increments up to the desired temperature. The response for a temperature change from $20^{\circ} \mathrm{C}-26^{\circ} \mathrm{C}$ is therefore obtained by adding the individual responses for each of the increments $20^{\circ} \mathrm{C}-22^{\circ} \mathrm{C}, 22^{\circ} \mathrm{C}-24^{\circ} \mathrm{C}$ and $24^{\circ} \mathrm{C}-26^{\circ} \mathrm{C}$ giving the true temperature response characteristic. This procedure results in a measurement error due to the 2 minute response decay periods, however this error is predictable and constant at each increment allowing remedial adjustment of the results.

\section{RESULTS AND DisCUSSION}

The low frequency admittance measurements for the temperature range $20^{\circ} \mathrm{C}$ to $40^{\circ} \mathrm{C}$ are shown in Fig. 2. The constant $B / f$ relationship over the $1-2 \mathrm{kHz}$ range at all temperatures indicates that the low frequency admittance is largely susceptive. Below $2 \mathrm{kHz}$ the effect of damping loss and inertial mass in the PZT film and brass substrate can therefore be considered negligible. It can therefore be inferred that since the influence of the inertial mass reduces with frequency that at frequencies below $1 \mathrm{kHz}$ the inertial mass will also have a negligible effect and that the $B / f$ relationship will remain linear down to DC. The low frequency capacitance $C_{t}$ is extracted from Fig. 2 by fitting a linear trend line at each temperature using Excel. $C_{t}$ is then determined from $\Delta B /$ $2 \pi \Delta f$. Fig. 3 shows the relationship between $C_{t}$ and temperature. $C_{t}$ increases approximately linearly by $13 \%$ over the temperature range $20^{\circ} \mathrm{C}$ and $40^{\circ} \mathrm{C}$, rising from $18.9 \mathrm{nF}$ to $21.4 \mathrm{nF}$. In addition, over the sensor typical operating range of $25^{\circ} \mathrm{C}$ to $35^{\circ} \mathrm{C}, C_{t}$ rises by $6 \%$. From (13) it can be seen that the sensitivity of the sensor is inversely proportional to $C_{t}$. The variation in $C_{t}$ will therefore have a significant effect on the performance of the sensor with the response being nonlinear and the sensitivity of the sensor reducing with increased temperature. The DC resistivity of the PZT film is also known to vary significantly with temperature over the range of interest [11]. However since the minimum measurement frequency of the Agilent B1500A analyser is $1 \mathrm{kHz}$ it is not possible to obtain a meaningful measurement of the DC resistivity using immitance measurements. The effect of resistivity can however be considered by introducing a complex clamped dielectric constant into (13) as outlined in section III- $B$. A pole will be introduced at $A / \rho l_{p} C_{t} \mathrm{rads}^{-1}$ where $\rho$ is the resistivity of the PZT film $(\Omega-\mathrm{m})$ resulting in a 

HERE TO EDIT) <

cut-on frequency of $A / 2 \pi \rho l_{p} C_{t} \mathrm{~Hz}$. Consequently an increase in $\rho$ will result in a reduction in the sensor cut-on frequency and vice-versa but will not affect the sensitivity.

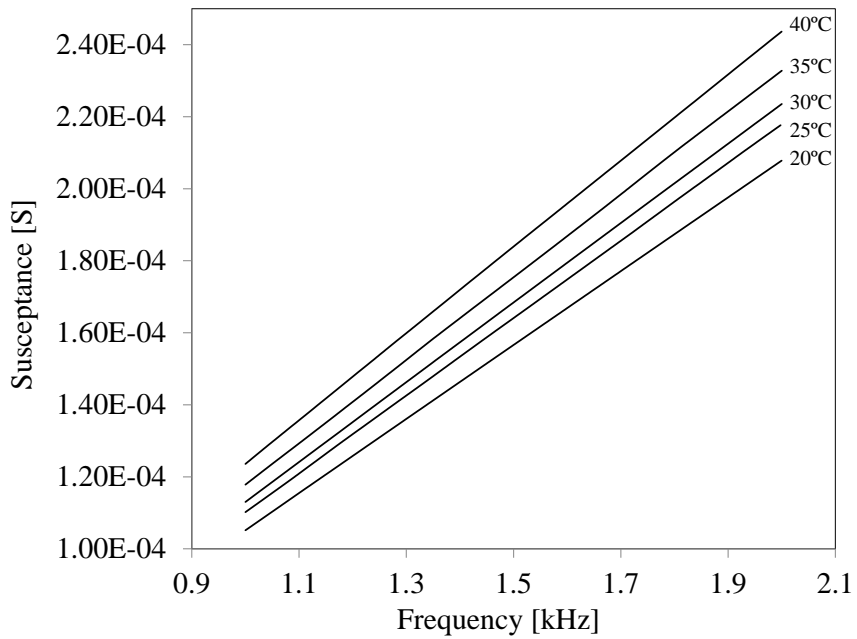

Fig. 2. Susceptance vs frequency from $20^{\circ} \mathrm{C}$ to $40^{\circ} \mathrm{C}$

The admittance results were compared with the BVD model defined above. At series resonance the term $\left(\omega L_{s}-1 / \omega_{s} C_{s}\right)$ in the denominator of $Y$ equals zero and the conductance $G$ reaches its peak value. The susceptance $B$ reduces to $\omega_{s} C_{0}$ where $\omega_{\mathrm{s}}$ is the series resonance frequency. The conductance $G$ and capacitance $B / \omega$ measurements around series resonance at $25^{\circ} \mathrm{C}$ for the first radial mode are shown in Fig. 4 with series resonance occurring at $94.84 \mathrm{kHz}$. The static capacitance $C_{o}$ is found directly by reading off the value of $B / \omega$ at the peak value of $G$.

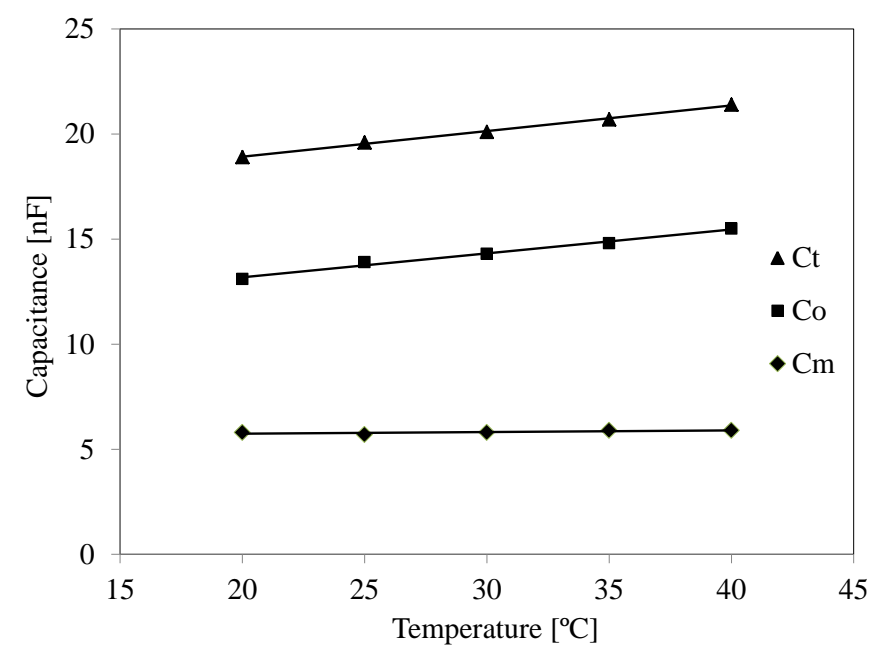

Fig. 3. Low frequency, clamped and mechanical equivalent capacitances vs temperature

Confirmation of $C_{o}$ can be made by noting that $C_{o}$ is also equal to the capacitance at $2 \omega_{a}$ equal to $B\left(2 \omega_{a}\right) / 2 \omega_{a}$ where $\omega_{a}$ is the anti-resonance frequency that occurs at maximum impedance [12]. At $25^{\circ} \mathrm{C}$ the anti-resonance frequency is measured at $93.54 \mathrm{kHz}$.

Fig. 4 shows the sensor capacitance and conductance with frequency at $25^{\circ} \mathrm{C}$. The static capacitance $C_{o}$ is measured at
$13.9 \mathrm{nF}$ at $25^{\circ} \mathrm{C}$. $C_{o}$ increases approximately linearly by $18 \%$ over the temperature range $20^{\circ} \mathrm{C}$ and $40^{\circ} \mathrm{C}$, rising from $13.1 \mathrm{nF}$ to $15.5 \mathrm{nF}$. Also, over the sensor typical operating range of $25^{\circ} \mathrm{C}$ to $35^{\circ} \mathrm{C}, C_{o}$ increases by $6.5 \%$. The values of $C_{o}$ over the temperature range $20^{\circ} \mathrm{C}$ and $40^{\circ} \mathrm{C}$ are given in Fig. 3 .

The low frequency electrical equivalent mechanical capacitance $C_{m}$ is calculated from $C_{m}=C_{t}-C_{0}$. The values of $C_{m}$ are given in Fig. 3. $C_{m}$ increases approximately linearly by only $1.7 \%$ over the temperature range $20^{\circ} \mathrm{C}$ and $40^{\circ} \mathrm{C}$, increasing from $5.8 \mathrm{nF}$ to $5.9 \mathrm{nF}$. However between $20^{\circ} \mathrm{C}$ and $25^{\circ} \mathrm{C}$ a drop of around $1.7 \%$ to $5.7 \mathrm{nF}$ is observed, rising again to $5.8 \mathrm{nF}$ at $30^{\circ} \mathrm{C}$. Given these small variations with temperature and taking into account a reasonable margin for measurement error, $C_{m}$ can be considered constant in the range $20^{\circ} \mathrm{C}$ to $40^{\circ} \mathrm{C}$.

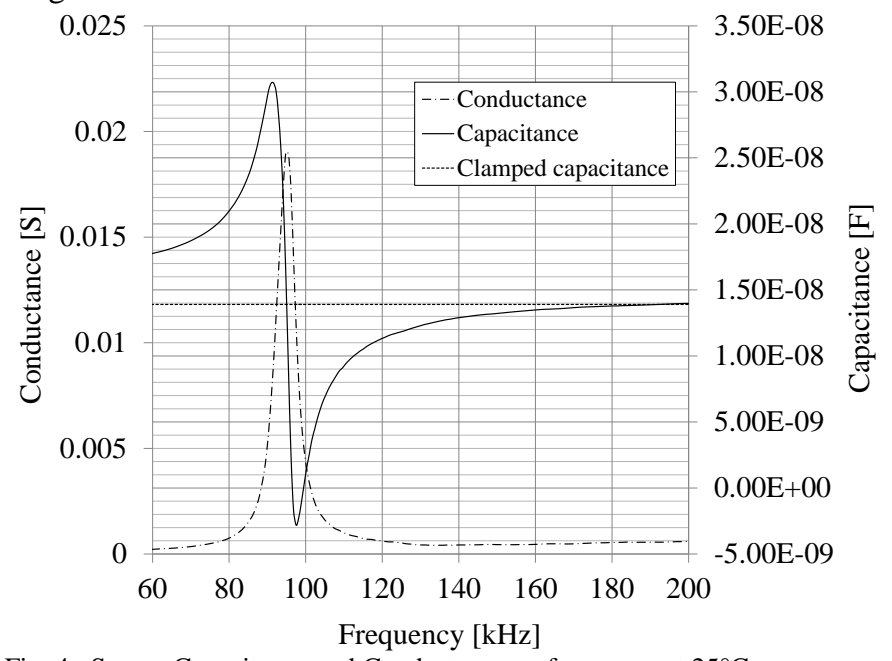

Fig. 4. Sensor Capacitance and Conductance vs frequency at $25^{\circ} \mathrm{C}$

It is therefore clear that the temperature dependency of the clamped capacitance $C_{0}$ via $\varepsilon_{33}^{S}$ will have a significant effect on the response of the sensor while the mechanical material constants are expected to have a relatively insignificant influence. Operation of the sensor using voltage mode signal conditioning will produce an amplifier response which is proportional to $V_{\text {out }}$ in (13) and is therefore dependent on $\varepsilon_{33}^{s}$. Alternatively, operation of the sensor using charge mode signal conditioning where the input impedance is low presents a solution since in the limit as as $R \rightarrow 0$ the amplifier response is proportional to $D_{s / c}$ given by (20) and is therefore independent of $\varepsilon_{33}^{S}$, In practice $R$ is finite and is largely due to the addition of two $100 \Omega$ series resistors to provide necessary additional electrostatic discharge protection at the amplifier input. As discussed in section IIIB, this additional resistance results in a high frequency attenuation and has a vanishingly small influence on the low frequency performance of the sensor. The impedance and time constant and therefore cut-on frequency of the sensor element is effectively replaced by that of the charge amplifier feedback network impedance. The output voltage of the amplifier is then proportional to a charge equal to $A D_{s / c}$ which is largely independent of $\varepsilon_{33}^{s}$. The unipolar charge amplifier is used for the measurement of the accumulative voltage. The forward gain stage is set to 0.5 to realise a measured sensitivity of $0.15 \mathrm{~V} /{ }^{\circ} \mathrm{C}$. A response of 
$0.15 \mathrm{~V} /{ }^{\circ} \mathrm{C}$ therefore requires a charge $/{ }^{\circ} \mathrm{C}$ of $0.15 C_{f}$ where $C_{f}$ is the $1.5 \mathrm{uF}$ feedback capacitance of a single charge amplifier stage. The effective pyroelectric coefficient $P_{\text {eff }}$ is therefore approximately $-0.15 C_{f} / A$ where $A$ is the area of the sensor element silver electrodes. The electrode radius is $9.1 \mathrm{~mm}$ and therefore $P_{e f f}$ is approximately $-865 \mu \mathrm{C} / \mathrm{m}^{2} /{ }^{\circ} \mathrm{C}$. The relative contribution to $P_{\text {eff }}$ of each term is now considered. Assuming that the PZT material is PZT5H, then using material data for PZT5H and brass from [11], [13], [14], and [15], the contributions are found to be:

$\frac{\beta\left(\varphi_{b}-\varphi_{p 1}\right) E_{b} A_{b}}{E_{b} A_{b}+\alpha\left(1-\mu_{b}\right) A_{p}}=-483 \mu \mathrm{C} / \mathrm{m}^{2} /{ }^{\circ} \mathrm{C}$ - Substrate clamping

$2 \varphi_{p 1} e_{31}+\varphi_{p 3} e_{33}=38 \mu \mathrm{C} / \mathrm{m}^{2} /{ }^{\circ} \mathrm{C}$ - Secondary effect

$P^{s}=-300$ to $-400 \mu \mathrm{C} / \mathrm{m}^{2} /{ }^{\circ} \mathrm{C}$ - Primary effect

$P_{\text {eff }}$ can thus be estimated at between -745 and $-845 \mu \mathrm{C}$ / $\mathrm{m}^{2} /{ }^{\circ} \mathrm{C}$. It is clear that the secondary pyroelectric effect is approximately $10 \%$ of the magnitude of the primary pyroelectric effect and that clamping by the substrate enhances the overall response of the sensing element by over $100 \%$. The contribution to $P_{e f f}$ of all three terms is therefore significant.

Fig. 5 shows the accumulative voltage response of the charge amplifier to incremental steps of $2^{\circ} \mathrm{C}$ from $20^{\circ} \mathrm{C}$ and $42^{\circ} \mathrm{C}$. The sensor exhibits a linear response over the measurements $20^{\circ} \mathrm{C} \rightarrow 42^{\circ} \mathrm{C}$ and $42^{\circ} \mathrm{C} \rightarrow 20^{\circ} \mathrm{C}$. A small round trip deviation of around $0.1 \mathrm{v}$ is evident. This is believed due to measurement error rather than an underlying physical process.

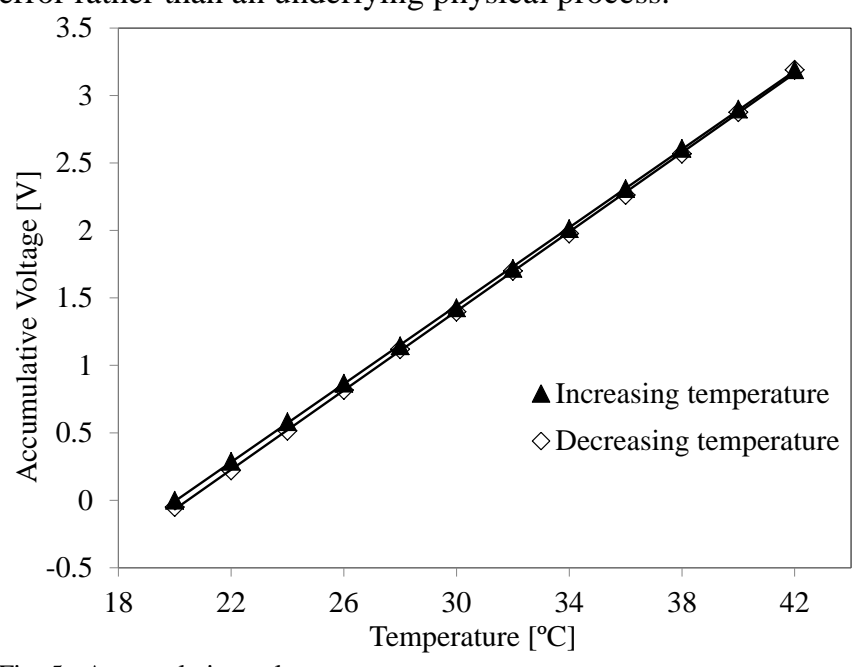

Fig. 5. Accumulative voltage vs temperature

The accuracy of the sensor is estimated by taking the mean of the amplifier response to each $2^{\circ} \mathrm{C}$ increment from $20^{\circ} \mathrm{C}$ to $42^{\circ} \mathrm{C}$ and taking the greatest positive and negative deviations from this mean as a percentage of the mean. These were found to be $+2.75 \%$ and $-3.25 \%$. A prudent estimate on accuracy can therefore be stated as $\pm 3.5 \%$. However since the temperature reference is obtained using a thermocouple integrated with the Peltier tile and PID controller, the validity of the estimate is therefore dependent on the accuracy of the thermocouple. With a typical accuracy of $\pm 1.1^{\circ} \mathrm{C}$ for the type $\mathrm{K}$ thermocouple used $\left( \pm 5.5 \%\right.$ at $20^{\circ} \mathrm{C}$ and $\pm 2.75 \%$ at $\left.40^{\circ} \mathrm{C}\right)$ over the temperature range of interest, the estimate of sensor accuracy must be treated with caution.
Sensor resolution will ultimately be limited by the digital interface used. The sensor output is digitised using an 8 bit Arduino Uno prototyping platform for temperature data logging and transmission. The 8 bit output gives 256 discrete levels and therefore for a temperature range of $20^{\circ} \mathrm{C}$ to $40^{\circ} \mathrm{C}$, the resolution can be stated as approximately $0.08^{\circ} \mathrm{C}$.

\section{CONCLUSION}

Models describing the low frequency response of the sensor under substrate clamping conditions have been developed and a prototype temperature sensor for e-Health applications based on the pyroelectric effect has been demonstrated. The sensor response under short-circuit conditions is demonstrated to be linear over the temperature range $20^{\circ} \mathrm{C}$ and $42^{\circ} \mathrm{C}$. A small $0.1 \mathrm{v}$ deviation at $20^{\circ} \mathrm{C}$ equivalent to $0.7^{\circ} \mathrm{C}$ in the round trip $20^{\circ} \mathrm{C} \rightarrow 42^{\circ} \mathrm{C} \rightarrow 20^{\circ} \mathrm{C}$ measurement requires further investigation to confirm measurement error. Further work is in progress to increase the sensor time constant and integrate the sensor into an Arduino Uno based wearable e-Health data acquisition and transmission system.

This project has received funding from the European Union's Horizon 2020 research and innovation programme under the Marie Skłodowska-Curie grant agreement No 734331.

\section{REFERENCES}

[1] L.E. Edsberg, R.E. Mates, and R.E. Baier, L Mark, "Mechanical characteristics of human skin subjected to static versus cyclic normal pressures, "Journal of rehabilitation research and development, vol. 36, no. 2, p. 133, Apr. 1999.

[2] J.T. Peery, W.R. Ledou, and G.K. Klute, "Residual-limb skin temperature in transtibial sockets," Journal of rehabilitation research and development, vol. 42, no. 2, p. 147, Mar. 2005.

[3] A.F.T. Mak, M. Zhang, and D.A. Boone, "State-of-the-art research in lower-limb prosthetic biomechanics-socket interface: a review," Journal of rehabilitation research and development, vol. 38, no. 2, p. 161, Mar. 2001.

[4] A.V. Afanas' ev, V. S. Lebedev, I. Ya Orlov, and A. E. Khrulev, "An infrared pyrometer for monitoring the temperature of materials in vacuum systems." Instruments and Experimental Techniques, vol. 44, no.2, pp. 275-278, March. 2001.

[5] C.C. Chang, and C. S. Tang. "An integrated pyroelectric infrared sensor with a PZT thin film," Sensors and Actuators A: Physical, vol. 65, no.23, pp. 171-174, March. 1998.

[6] R.G. Köhler, R., G. Suchaneck, P. Padmini, T. Sandner, G. Gerlach, and G. Hofmann, "RF-sputtered PZT thin films for infrared sensor arrays," Ferroelectrics, vol. 225, no.1, pp. 57-66, March. 1999.

[7] M.B. Silver-Thorn, J.W.Steege, and D.S. Childress, "A review of prosthetic interface stress investigations," Journal of rehabilitation research and development, vol. 33, no.3, p. 253, Jul. 1996.

[8] N. Mathur, I. Glesk, and A. Buis, "Skin temperature prediction in lower limb prostheses," IEEE Journal of Biomedical and Health informatics, vol. 20, no.1, pp. 158-165, Jan. 2016.

[9] K. Ghoseiri, Y.P. Zheng, A.K. Leung, M. Rahgozar, G. Aminian, T.H. Lee, and M.R. Safari, "Temperature Measurement and Control System for Transtibial Prostheses: Functional Evaluation," Assistive Technology, pp. 1-8, Nov. 2016.

[10] M.W. Hooker, "Properties of PZT-Based piezoelectric Ceramics between-150 and 250C," National Aeronautics and Space Administration. Tech. Rep. CR-1998-208708, Sept. 1998.

[11] S. Sherrit, B. K. Mukherjee, (2007, Nov.) Characterisation of piezoelectric materials for transducers" Arxiv [online] Available: http://arxiv.org/ftp/arxiv/papers/0711/0711.2657.pdf.

[12] https://www.scribd.com/doc/34864965/PZT-Material-Properties

[13] http://www.piezo.com/prodmaterialprop.html

[14] http://www.engineeringtoolbox.com/ 